\title{
Score risk model for predicting severe fever with thrombocytopenia syndrome mortality \\ (a) CrossMark
}

\author{
Li Wang ${ }^{*}$, Zhiqiang Zou ${ }^{\dagger}$, Chunguo Hou, Xiangzhong Liu, Fen Jiang and Hong Yu
}

\begin{abstract}
Background: Severe fever with thrombocytopenia syndrome (SFTS) is an emerging epidemic infectious disease with high mortality in East Aisa, especially in China. To predict the prognosis of SFTS precisely is important in clinical practice.

Methods: From May 2013 to November 2015, 233 suspected SFTS patients were tested for SFTS virus using RT-PCR. Cox regression model was utilized to comfirm independent risk factors for mortality. A risk score model for mortality was constructed based on regression coefficient of risk factors. Log-rank test was used to evaluate the significance of this model.

Results: One hundred seventy-four patients were confirmed with SFTS, of which 40 patients died (23\%). Baseline age, serum aspartate aminotransferase (AST) and serum creatinine ( $\mathrm{s} C r$ ) level were independent risk factors of mortality. The area under ROC curve (AUCS) of these parameters for predicting death were $0.771,0.797$ and 0.764 , respectively. And hazard ratio (HR) were 1.128, 1.002 and 1.013, respectively. The cutoff value of the risk model was 10. AUC of the model for predicting mortality was 0.892 , with sensitivity and specificity of 82.5 and $86.6 \%$, respectively. Log-rank test indicated strong statistical significance $\left(x^{2}=88.35, p<0.001\right)$.
\end{abstract}

Conclusions: This risk score model may be helpful to predicting the prognosis of SFTS patients.

Keywords: Risk model, Severe fever with thrombocytopenia syndrome, Prediction, Mortality

\section{Background}

Severe fever with thrombocytopenia syndrome (SFTS) is an emerging epidemic infectious disease in China, Korea and Japan caused by a novel bunyavirus, SFTS virus (SFTSV). It has become an important public health threat in Asia, especially in China. The national surveillance data from 2010 to 2013 in China showed that the incidence of SFTS and its epidemic areas are growing, but the case fatality rate (CFR) has steadily declined [1]. The major clinical symptoms of SFTS include fever, thrombocytopenia, leukocytopenia, gastrointestinal symptoms and various other systemic manifestations including muscular symptoms, neurological disorders and coagulopathy $[2,3]$. Neurological symptoms were strongly associated with death, even though they occurred during the first week after disease onset [4]. Other factors associated with death include viral load [5-8], advanced

\footnotetext{
*Correspondence: liliwang2200@163.com

${ }^{\dagger}$ Equal contributors

Infectious Disease Hospital of Yantai, 62 Huanshan Road, Zhifu district, Yantai, Shandong 264001, China
}

age, serum aspartate aminotransferase (AST), lactate dehydrogenase $(\mathrm{LDH})$ and creatine kinase $(\mathrm{CK})$ levels, and decreased lymphocyte percentages [7-12].

We and others [13] have found in clinical practice that though some patients had similar symptoms at admission, including altered consciousness and multiple organ dysfunctions and received similar therapy, their clinical courses were quite different. However, several patients who died afterwards did not exhibit neurological disorders at admission. Thus it is important to predict the prognosis of the patients in clinical practice.

Though the viral load is a predictor of morbidity [13], in contrast to routine laboratory examination, which is quickly and easily performed, the level of SFTS viral RNA requires several days to determine, and can only be conducted at a limited number of local disease control institutes. Furthermore, the viral RNA only becomes detectable in most cases at three days and reaches maximum level at six days after disease onset. In $55.6 \%$ of the patients, viral RNA was undetectable at hospital 
admission [8]. In this study, we established a risk score model for predicting mortality of SFTS patients based on their clinical and demographic characteristics, and routine laboratory tests including biochemical, hematological parameters and coagulation variables.

\section{Methods}

\section{Clinical samples and laboratory testing}

Patients with clinical manifestations of suspected SFTS, including fever (body temperature $>38.0{ }^{\circ} \mathrm{C}$ prior to admission or at admission), thrombocytopenia, leukocytopenia and gastrointestinal symptoms were tested for SFTSV via reverse transcription-polymerase chain reaction (RT-PCR) using total RNA extracted from a peripheral blood sample [14] at local institute for disease preventation and control. The results are given as positive or negative afterwards for clinicians to confirm SFTSV infection. Clinical history and manifestation, physical examination, routine biochemical, hematological and coagulation results at enrollment were retrospectively collected from confirmed SFTS patients. Hematological parameters were detected using an XT1800I blood cell analyzer and biochemical parameters using a CI16200 blood biochemical automatic analyzer. Neurological symptoms included: limb shaking, lower jaw shaking, dysarthria and discordance of consciousness.

Parameters which entered in Cox univariate regression analysis included: age, gender, body temperature, time intervals between symptom onset to admission, hematological parameters, biochemical parameters, coagulation indicators and neurological symptom.

This study was conducted according to the Helsinki II Declaration and approved by the ethics committee at the National Institute of Parasitic Diseases, Chinese Center for Disease Control and Prevention. Written informed consent was obtained from the patients.

\section{Statistical analysis}

Statistical analyses were performed using the SPSS, version 16.0, software (IBM, Armonk, NY, USA). MannWhitney $U$ test was used for variables with an abnormal distribution between the survival and death groups. Cox univariate and multivariate regression analysis was applied to determine the risk factors among parameters at admission that were associated with mortality of SFTS patients. Factors with $p<0.1$ in univariate analysis were included in Cox multivariate regression analysis. Risk score model was established based on the acquired regression coefficients of the determined risk factors to predict mortality. Criterion for factors that include in the regression model was that the factors present in the last step of forward conditional with $p<0.05$ and Hazard ratios (HR) $>1.0$. Receiver-operating characteristic (ROC) curves and area under the curve (AUC) were constructed to assess the predictive power of the model. Patients were divided into high-risk and low-risk groups according to cutoff values obtained from ROC analysis. The KaplanMeier survival analysis was used to compare the cumulative risk for death in the two groups, and the significance of difference was tested with the log-rank test. $x^{2}$ test was utilized to compare mortality rate between patients with above and less than cutoff value of risk model. $p<0.05$ was considered to be statistically significant.

\section{Results}

Demographic and clinical characteristics of SFTS patients Among the 233 suspected cases with fever, thrombocytopenia, fatigue and diarrhea admitted to our hospital from May 2013 to November 2015, 174 were finally diagnosed as SFTS by detecting SFTSV using RT-PCR. Forty patients (23\%) subsequently died. The survival group included 63 women (47.0\%) and 71 men (53.0\%), while the non-survival group comprised of 19 women (47.5\%) and 21 men (52.5\%). Gender composition was identical in the two groups. The median age was 64 (range 28-84) years, and was significantly higher among those who died (range 52-84 years; $p<0.001$ ).

The major baseline clinical symptoms and findings included fever $(n=102,58.6 \%)$, fatigue $(n=120,69.0 \%)$, loss of appetite $(n=86,50 \%)$ diarrhea $(n=12,6.9 \%)$, superficial lymphadenopathy $(n=72,41.4 \%)$, neutropenia $(n=110,63.2 \%)$, thrombocytopenia $(n=174,100 \%)$ and neurological symptoms $(n=63,36.2 \%)$. Among those who survived, 38 cases manifested neurological symptoms. Nine cases had severe neurological symptoms included abnormal level of consciousness. Others exhibited mainly limb shaking. Among those patients who have died, 25 cases had neurological symptoms (60\%) at admission and other developed central neurological symptoms primarily discordance of consciousness and coma within 3-7 days. Ages of those with severe neurological symptoms who survived are younger than those who died $(62.3 \pm 7.8$ vs. $70.9 \pm 9.5, p<0.05)$.

Body temperature at admission in surviving and fatal cases $\left(37.4 \pm 0.98^{\circ} \mathrm{Cvs} 37.5 \pm 1.1{ }^{\circ} \mathrm{C}, p>0.05\right)$ were comparable. The time intervals between symptom onset to admission were also similar between the two groups $(6.5 \pm 3.3$ vs $5.6 \pm 1.9, p>0.05)$. Hospitalization duration was longer in patients who recovered than those who died $(13.0 \pm 5.5$ vs $3.4 \pm 2.4, p<0.001)$.

\section{Comparison of biochemical and hematological parameters in surviving and fatal SFTS cases}

For the biochemical parameters, levels of alanine aminotransferase (ALT), AST, total bilirubin (TB), high-sensitivity C-reactive protein (hs-CRP), blood urea nitrogen (BUN), serum creatinine $(\mathrm{sCr})$, troponin (Ctnl), CK, hydroxybutyrate dehydrogenase $(\mathrm{HBDH}), \mathrm{LDH}$, prothrombin 
time (PT) and international normalized ratio (INR) were dramatically increased in the fatal cases. While the prothrombin time activity percentage (PTA) was markedly decreased. Concentrations of serum potassium $\left(\mathrm{K}^{+}\right)$and phosphorus $\left(\mathrm{P}^{3+}\right)$ in the fatal cases were significantly elevated, whereas sodium $\left(\mathrm{Na}^{+}\right)$, calcium $\left(\mathrm{Ca}^{2+}\right)$ and hydrocarbonate $\left(\mathrm{HCO}_{3-}\right)$ were notably reduced.

For the hematological parameters, white cell blood (WBC) count, neutrophil (NEU) count, neutrophil/ lymphocyte $(\mathrm{N} / \mathrm{L})$ ratio, mean corpuscular volume (MCV) and red blood volume distributing width (RDW) in the fatal cases were remarkably increased. Red blood cell (RBC) count, lymphocyte (LYM) count, PLT count and thrombocytocrit (PCT) were prominently decreased. These results are shown in Tables 1 and 2 .

Table 1 Comparison of biochemical parameters in survivals and non-survivals of STFS patients $(\bar{x} \pm$ SD)

\begin{tabular}{|c|c|c|c|}
\hline Parameters & Survival & Non-survival & $p$ \\
\hline $\bar{N}$ & 134 & 40 & \\
\hline Male/female & $71 / 63$ & $21 / 19$ & $>0.05$ \\
\hline Age (year) & $61.2 \pm 9.9$ & $71 \pm 9.5$ & $<0.001$ \\
\hline Hospital stays (day) & $13.0 \pm 5.5$ & $3.4 \pm 2.4$ & $<0.001$ \\
\hline Body temperature $\left({ }^{\circ} \mathrm{C}\right)$ & $37.4 \pm 0.98$ & $37.5 \pm 1.0$ & $>0.05$ \\
\hline $\operatorname{ALT}(\mathrm{U} / \mathrm{L})$ & $95.4 \pm 86.7$ & $191.8 \pm 148.8$ & $<0.001$ \\
\hline AST $(U / L)$ & $210.3 \pm 219.0$ & $713.4 \pm 572.9$ & $<0.001$ \\
\hline $\mathrm{TB}(\mu \mathrm{mol} / \mathrm{L})$ & $11.6 \pm 5.0$ & $19.0 \pm 13.4$ & $<0.001$ \\
\hline $\mathrm{HCO}-(\mathrm{mmol} / \mathrm{L})$ & $24.1 \pm 3.0$ & $22.6 \pm 5.5$ & $<0.05$ \\
\hline $\mathrm{Ca} 2+(\mathrm{mmol} / \mathrm{L})$ & $1.96 \pm 0.13$ & $1.86 \pm 0.17$ & $<0.01$ \\
\hline $\mathrm{P} 3+(\mathrm{mmol} / \mathrm{L})$ & $0.93 \pm 0.25$ & $1.04 \pm 0.31$ & $<0.05$ \\
\hline $\mathrm{Hs}-\mathrm{CRP}(\mathrm{mg} / \mathrm{L})$ & $9.6 \pm 11.6$ & $18.3 \pm 15.7$ & $<0.001$ \\
\hline Dimer (ng/L) & $5.2 \pm 10.5$ & $14.4 \pm 22.4$ & $<0.001$ \\
\hline BUN (mmol/L) & $4.9 \pm 2.4$ & $10.1 \pm 5.9$ & $<0.001$ \\
\hline $\mathrm{SCr}(\mu \mathrm{mol} / \mathrm{L})$ & $64.1 \pm 16.3$ & $115.1 \pm 79.3$ & $<0.001$ \\
\hline UA ( $\mu \mathrm{mol} / \mathrm{L})$ & $286.8 \pm 117.6$ & $430.4 \pm 192.2$ & $<0.001$ \\
\hline Ctnl (ng/mL) & $8.5 \pm 93.6$ & $1.2 \pm 3.6$ & $<0.001$ \\
\hline CK (U/L) & $916.7 \pm 1424.4$ & $2372.0 \pm 3680.1$ & $<0.001$ \\
\hline CK-MB (U/L) & $47.8 \pm 54.8$ & $85.5 \pm 78.5$ & $<0.001$ \\
\hline $\mathrm{HBDH}(\mathrm{U} / \mathrm{L})$ & $4846.8 \pm 326.2$ & $967.1 \pm 848.2$ & $<0.001$ \\
\hline $\mathrm{LDH}(\mathrm{U} / \mathrm{L})$ & $804.1 \pm 811.8$ & $1989.3 \pm 1862.1$ & $<0.001$ \\
\hline PT (s) & $12.2 \pm 1.3$ & $13.6 \pm 2.0$ & $<0.01$ \\
\hline PTA (\%) & $107.1 \pm 18.9$ & $89.1 \pm 17.3$ & $<0.01$ \\
\hline INR & $0.98 \pm 0.11$ & $1.09 \pm 0.17$ & $<0.01$ \\
\hline
\end{tabular}

ALT alanine aminotransferase, AST aspartate aminotransferase, $h s-C R P$ highsensitivity C-reactive protein, $B U N$ blood urea nitrogen, $\mathrm{s} C r$ serum creatinine, $C$ tnl troponin, $C K$ creatine kinase, $H B D H$ hydroxybutyrate dehydrogenase, $L D H$ lactate dehydrogenase, $P T$ prothrombin time, PTA prothrombin time activity percentage, INR international normalized ratio
Table 2 Comparison of hematological parameters in survivals and non-survivals of STFS patients $(\bar{x} \pm$ SD)

\begin{tabular}{lccc}
\hline Parameters & Survival & Death & $p$ \\
\hline WBC $\left(\times 10^{9} / \mathrm{mL}\right)$ & $4.02 \pm 2.71$ & $4.14 \pm 2.68$ & $>0.05$ \\
NEU $(\%)$ & $58.3 \pm 17.1$ & $68.5 \pm 16.7$ & $<0.01$ \\
LYM (\%) & $30.2 \pm 13.4$ & $21.6 \pm 11.0$ & $<0.01$ \\
N/L ratio & $2.77 \pm 2.50$ & $4.74 \pm 3.77$ & $<0.01$ \\
MON (\%) & $10.78 \pm 6.62$ & $8.47 \pm 6.36$ & $<0.05$ \\
EOS (\%) & $0.08 \pm 1.07$ & $1.25 \pm 1.73$ & $>0.05$ \\
RBC $\left(\times 10^{12} / \mathrm{mL}\right)$ & $4.6 \pm 0.53$ & $4.39 \pm 0.45$ & $<0.05$ \\
Hb $(\mathrm{g} / \mathrm{L})$ & $138.7 \pm 18.1$ & $138.5 \pm 14.7$ & $>0.05$ \\
HCT $(\%)$ & $39.1 \pm 4.5$ & $38.6 \pm 4.3$ & $>0.05$ \\
MCV $(\mathrm{fL})$ & $85.0 \pm 5.6$ & $88.0 \pm 6.4$ & $<0.01$ \\
RDW $(\%)$ & $13.3 \pm 1.3$ & $14.5 \pm 7.9$ & $<0.05$ \\
PLT $\left(\times 10^{9} / \mathrm{mL}\right)$ & $60.8 \pm 35.6$ & $40.2 \pm 13.7$ & $<0.01$ \\
PCT & $0.07 \pm 0.04$ & $0.05 \pm 0.03$ & $<0.01$ \\
MPV $(\mathrm{fL})$ & $11.0 \pm 1.0$ & $10.8 \pm 2.1$ & $>0.05$ \\
\hline
\end{tabular}

WBC white blood cell, NEU neutrophil, $L Y M$ lymphocyte, N/L ratio neutrophil/ lymphocyte ratio, MON monocyte, EOS eosnophils, $R B C$ red blood cell, $H b$ hemoglobin, $H C T$ hematocrit, $M C V$ mean corpuscular volume, RDW red cell distribution width, PLT platelet count, $P C T$ thrombocytocrit, MPV mean platelet volume

\section{Establishment of risk score model}

Cox regression model analysis showed that baseline parameters, including age, serum AST level and sCr level were independent risk factors of mortality. HR (95\% CI), and mean survival times of these factors are shown in Table 3. Age is the most remarkable risk factor among these parameters with $\mathrm{HR}=1.128$.

The cutoff values and area under ROC curve (AUCs) of these parameters for predicting death are included in Table 4. AST level has the highest predictive value among these factors. Based on regression coefficients of multivariate Cox regression analysis of these factors, we constructed a risk score model for the prediction of mortality in SFTS patients.

$$
\begin{aligned}
\operatorname{Model}(\mathrm{M})= & 0.002 \times \mathrm{AST}+0.121 \times \mathrm{AGE} \times \\
& +0.013 \times \mathrm{sCr}
\end{aligned}
$$

The cutoff value of the score model was 10 . Patients with $>10$ were at high-risk of mortality. AUC of the model for predicting mortality was 0.892 , with sensitivity and specificity of 82.5 and $86.6 \%$, respectively. Mean survival time of patients with $M>10$ is shorter than those with $\mathrm{M} \leq 10$. Log-rank test indicated strong statistical significance $\left(x^{2}=88.35, p<0.001\right)$. Data are shown in Tables 3 and 4 . ROCs and survival curves are depicted in Figs. 1 and 2. Of those with $M \leq 10(n=123), 7$ cases died (5.7\%). And of those with $M>10(n=51), 33$ cases 
Table 3 Hazard ratio (HR) and means survival time of factors independently associated with mortality

\begin{tabular}{|c|c|c|c|c|c|}
\hline \multirow[t]{2}{*}{ Parameters } & \multirow[t]{2}{*}{$\mathrm{HR}(95 \% \mathrm{Cl})$} & \multirow[t]{2}{*}{ Cutoff value } & \multirow[t]{2}{*}{ Mean time (days) $(95 \% \mathrm{Cl})$} & \multicolumn{2}{|c|}{ Log Rank } \\
\hline & & & & $\overline{x^{2}}$ & $p$ \\
\hline \multirow[t]{2}{*}{ Age (year) } & $1.128(1.071,1.189)$ & $\leq 66$ & $28.8 \pm 0.86(27.2,30.6)$ & 28.3 & $<0.001$ \\
\hline & & $>66$ & $17.9 \pm 1.72(14.5,21.2)$ & & \\
\hline \multirow[t]{2}{*}{ AST (U/L) } & $1.002(1.001,1.003)$ & $\leq 437$ & $28.6 \pm 0.8(27.0,30.0)$ & 55.1 & $<0.001$ \\
\hline & & $>437$ & $11.2 \pm 1.76(7.7,14.6)$ & & \\
\hline \multirow[t]{2}{*}{$\mathrm{s} C r(\mu \mathrm{mmol} / \mathrm{L})$} & $1.013(1.006,1.020)$ & $<70$ & $23.7 \pm 0.66(22.4,25)$ & 35.7 & $<0.001$ \\
\hline & & $\geq 70$ & $17.4 \pm 1.96(13.5,21.2)$ & & \\
\hline \multirow[t]{2}{*}{ M } & & $\leq 10$ & $30.3 \pm 0.62(29.1,31.5)$ & 88.4 & $<0.001$ \\
\hline & & $>10$ & $12.0 \pm 1.83(8.4,15.6)$ & & \\
\hline
\end{tabular}

died (64.7\%). Mortality rates between two groups had strong significant difference $\left(x^{2}=70.9, p<0.001\right)$.

\section{Prospective validation}

The cutoff value of $\mathrm{M}(=10)$ was prospectively validated in a cohort of SFTS patients $(n=67)$ enrolled in our hospital this year. Of whom, 51 cases survived (76.1\%) and 16 died (23.9\%). Of those patients with $M \leq 10$ $(n=39,58.2 \%), 36$ cases survived $(92.4 \%)$ and 3 cases died $(7.6 \%)$. Of those with $M>10(n=28,41.8 \%), 13$ cases died (46.4\%) and 15 cases survived (53.6\%). Mortality rates between these two groups had significant difference $\left(x^{2}=13.5, p<0.001\right)$.

\section{Discussion}

In this study, we established a risk model for the prediction of mortality of SFTS patients that showed relatively high predictive value with high sensitivity and specificity. The model contains 3 high-risk factors including age, serum AST level and sCr level, which have moderate predictive values for mortality when used independently. Numerous observational studies have shown that neurological symptoms were strongly associated with death. In this study, though not all the fatal patients have neurological symptoms at admission, all cases developed neurological symptoms afterwards. While, results showed that neurological symptoms was not an independent risk factor. The reason may be that neurological symptoms in survival patients were relative mild and the symptoms were severe in the death group. Ages of those critical patients with severe neurological symptoms who survived were younger than those who died. As we shown, age is the highest risk factor of the parameters. Elevated serum AST and sCr level may represent multi-organ dysfunction.

Other factors such as time intervals between symptom onset and treatment initiation, and therapeutic methods may also influence the prognosis of the patients. A delayed diagnosis of SFTS could affect the prognosis of the patients [4].

Other study showed that cytokines, such as interleukine (IL)-1, IL-6, IL-10, granulocyte colony stimulating factor (G-CSF), interferon inducible protein-10 (IP-10) and monocyte chemoattractant protein (MCP-1) were profoundly elevated in fatal cases, and their levels correlated with various clinical parameters [10]. While, the measurements of several serum cytokine levels together are too expensive to be performed by all healthcare facilities in China, so it is unsuitable to be used to predict the clinical prognosis.

Though only $58.6 \%$ patients had fever at adimissiom, and body temperature was not associated with prognosis, all patients had fever prior admission or body

Table 4 Cut-off values, AUCs of age and biochemical parameters for the prediction of mortality of SFTS patients with sensitivity and specificity

\begin{tabular}{|c|c|c|c|c|c|c|c|c|}
\hline Parameters & cutoff value & AUCs $(95 \% \mathrm{Cl})$ & SEN (\%) & SPE (\%) & PPV (\%) & NPV (\%) & LR+ & $\overline{\text { LR- }}$ \\
\hline Age (year) & 66 & $0.771(0.701,0.831)$ & 75 & 70.2 & 42.9 & 90.4 & 2.51 & 0.36 \\
\hline AST (U/L) & 437 & $0.797(0.730,0.854)$ & 60 & 90.3 & 64.9 & 88.3 & 6.18 & 0.44 \\
\hline $\mathrm{sCr}(\mu \mathrm{mml} / \mathrm{L})$ & 70 & $0.764(0.692,0.827)$ & 71.8 & 77.6 & 50.0 & 89.8 & 3.21 & 0.36 \\
\hline M & 10 & $0.892(0.829,0.955)$ & 82.5 & 86.6 & 64.7 & 94.3 & 6.14 & 0.20 \\
\hline
\end{tabular}

AUC area under, $R O C$ curve, SEN sensitivity, SPE specificity, PPV positive predictive value, NPV negative predictive value, $L R+$ positive likelihood ratio, $L R$ - negative likelihood ratio 


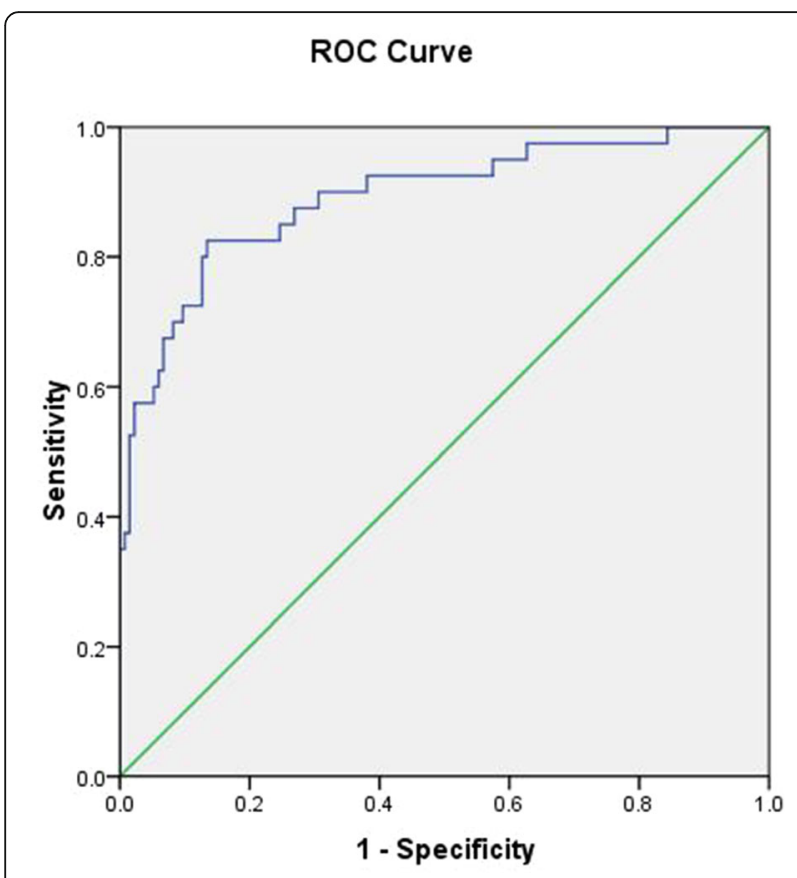

Fig. 1 ROC curve and area under ROC curve of risk score model for the prediction of mortality of SFTS patients

temperature elevated after admission. Fever and fatigue were the main chief complaint of SFTS patients. Though the fatality rate of SFTS in China has fallen from first reported $30 \%$ to present $12 \%$ owing to increased reporting, advances in diagnostic procedures and improved clinicians' skills $[5,15,16]$. The risk factors for human infection with SFTSV were confirmed $[15,17]$ and national intervention programs were performed, including promoting public awareness, establishing sentinel hospitals and improving clinicians' skills [2,18-20]. Yet, the number of fatal cases increases annually in China.

Except for new treatment methods tried in a few therapies with few patients in Japan and South Korea [21-23], supportive care remains the standard treatment for SFTS. Therefore, accurate prediction of the prognosis may help clinicians to perform intervention measures in advance, control the disease progression and improve the prognosis.

In the prospective study, we showed that the model is more suitable for the prediction of survival than for mortality. The model we constructed will be helpful for the prediction of SFTS prognosis. And the results will be further prospectively validated by a more larger cohort of patients.

This study had several limitations. Firstly, we had no quantitative results of the virus RNA and only had the positive results. Secondly, patients with subclinical or minor infection signs who sought medical care were perhaps not tested for SFTSV. Thirdly, some patients with mild manifestations may have been excluded due to their low/undetectable viral loads. All these factors influence the accuracy of registered incidence and mortality.

\section{Conclusions}

In summary, the risk score model that we established is useful for predicting death in patients with SFTS and may be applicable in clinical practice.

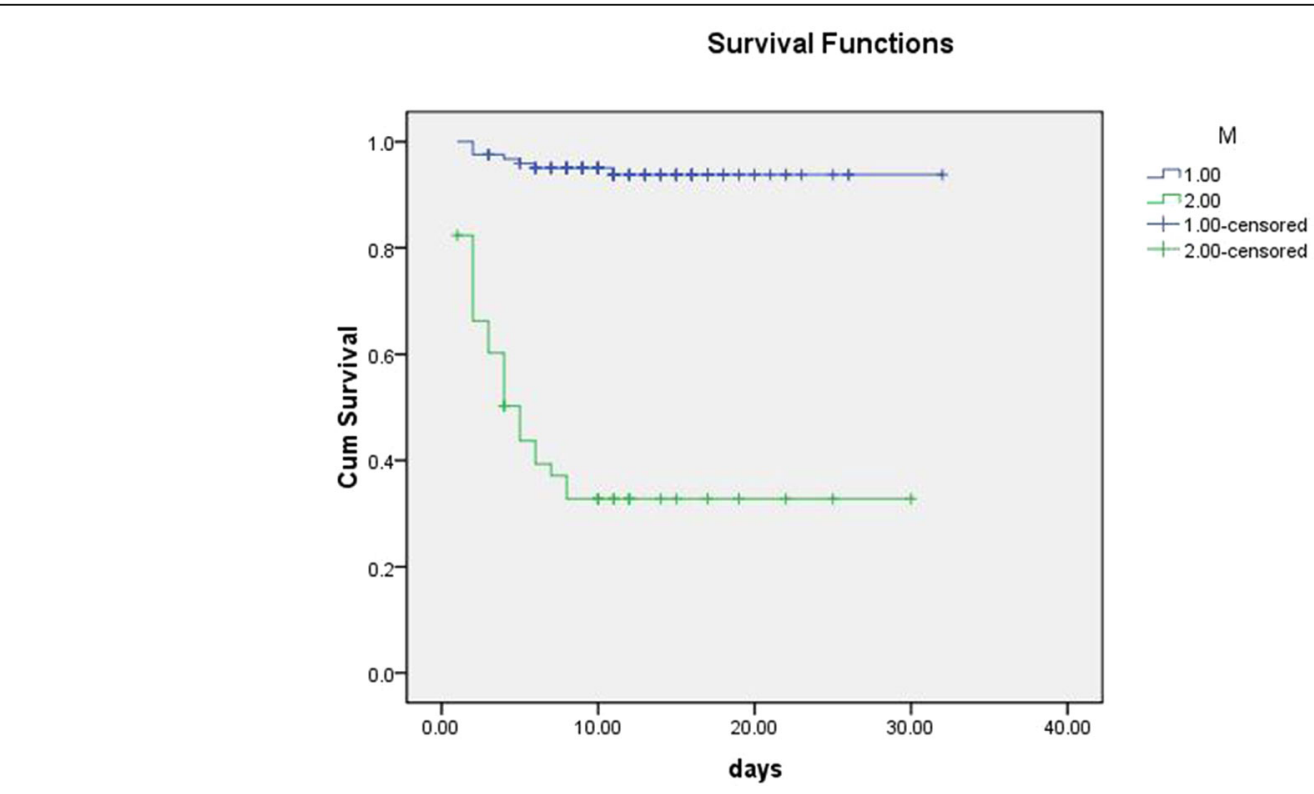

Fig. 2 Survival curve of SFTS patients based on risk score model 


\section{Abbreviations}

ALT: Alanine aminotransferase; AST: Aspartate aminotransferase; AUCs: Area under ROC curve; BUN: Blood urea nitrogen; CFR: Case fatality rate; CK: Creatine kinase; G-CSF: Granulocyte colony stimulating factor; HBDH: Hydroxybutyrate dehydrogenase; HR: Hazard ratios; hs-CRP: Highsensitivity C-reactive protein; IL-1: Interleukine-1; INR: International normalized ratio; IP-10: Interferon inducible protein-10; LDH: Lactate dehydrogenase; LYM: Lymphocyte; MCP-1: Monocyte chemoattractant protein; MCV: Mean corpuscular volume; N/L: Neutrophil/lymphocyte; NEU: Neutrophil; PLT: Platelet; PT: Prothrombin time; PTA: Prothrombin time activity; ROC: Receiver-operating characteristic; RT-PCR: Reverse transcription-polymerase chain reaction; sCr: Serum creatinine; SFTS: Severe fever with thrombocytopenia syndrome; TB: Total bilirubin; WBC: White blood cell

\section{Acknowledgements}

We are grateful for the laboratory technicians in the institute for disease preventation and control of Yantai city for their testing of SFTSV RNA.

\section{Availability of data and materials}

All relevant data are within the paper.

\section{Authors' contributions}

LW and ZZ conceived and designed the research. $\mathrm{CH}, \mathrm{XL}$, JF and HY collected clinical samples. LW performed the data analysis and modeling. LW wrote the manuscript. All authors reviewed the manuscript. All authors read and approved the final manuscript.

\section{Competing interests}

The authors declare that they have no competing interests.

\section{Consent for publication}

The written informed consent was obtained from every participant in this study.

\section{Ethics approval and consent to participate}

This study was conducted according to the Helsinki II Declaration and approved by the ethics committee at the National Institute of Parasitic Diseases, Chinese Center for Disease Control and Prevention.

Received: 29 June 2016 Accepted: 10 December 2016

Published online: 07 January 2017

\section{References}

1. Liu K, Zhou H, Sun RX, Yao HW, Li Y, Wang LP, et al. A national assessment of the epidemiology of severe fever with thrombocytopenia syndrome. China Sci Rep. 2015:5:9679.

2. Guo CT, Lu QB, Ding SJ, Hu CY, Hu JG, Wo Y, et al. Epidemiological and clinical characteristics of severe fever with thrombocytopenia syndrome (SFTS) in China: an integrated data analysis. Epidemiol Infect. 2016;144 1345-54.

3. Li DX. Severe fever with thrombocytopenia syndrome: a newly discovered emerging infectious disease. Clin Microbiol Infect. 2015;21:614-20.

4. Shin J, Kwon D, Youn SK, Park JH. Characteristics and Factors Associated with Death among Patients Hospitalized for Severe Fever with Thrombocytopenia Syndrome, South Korea, 2013. Emerg Infect Dis. 2015;21: 1704-10.

5. Gai ZT, Zhang Y, Liang MF, Jin C, Zhang S, Zhu CB, et al. Clinical progress and risk factors for death in severe fever with thrombocytopenia syndrome patients. J Infect Dis. 2012;206:1095-102.

6. Liu Q, He B, Huang SY, Wei F, Zhu XQ. Severe fever with thrombocytopenia syndrome, an emerging tick-borne zoonosis. Lancet Infect Dis. 2014;14:763-72.

7. Zhang YZ, He YW, Dai YA, Xiong Y, Zheng H, Zhou DJ, et al. Hemorrhagic fever caused by a novel Bunyavirus in China: pathogenesis and correlates of fatal outcome. Clin Infect Dis. 2012;54:527-33.

8. Yang ZD, Hu JG, Lu QB, Guo CT, Cui N, Peng W, et al. The prospective evaluation of viral loads in patients with severe fever with thrombocytopenia syndrome. J Clin Virol. 2016;78:123-8.

9. Ding S, Niu G, Xu X, Li J, Zhang X, Yin H, et al. Age is a critical risk factor for severe fever with thrombocytopenia syndrome. PLoS One. 2014;9:e111736.
10. Sun $Y$, Jin C, Zhan F, Wang X, Liang M, Zhang Q, et al. Host cytokine storm is associated with disease severity of severe fever with thrombocytopenia syndrome. J Infect Dis. 2012;206:1085-94.

11. Deng B, Zhou B, Zhang S, Zhu Y, Han L, Geng Y, et al. Clinical features and factors associated with severity and fatality among patients with severe fever with thrombocytopenia syndrome Bunyavirus infection in Northeast China. PLoS One. 2013;8:e80802.

12. Cui F, Cao HX, Wang L, Zhang SF, Ding SJ, Yu XJ, et al. Clinical and epidemiological study on severe fever with thrombocytopenia syndrome in Yiyuan County, Shandong Province. China Am J Trop Med Hyg. 2013;88: 510-2.

13. Kitao A, leki R, Takatsu H, Tachibana Y, Nagae M, Hino T, et al. Severe fever with thrombocytopenia syndrome presenting as hemophagocytic syndrome: two case reports. Springerplus. 2016;5:361

14. Yoshikawa T, Fukushi S, Tani H, Fukuma A, Taniguchi S, Toda S, et al. Sensitive and specific PCR systems for detection of both Chinese and Japanese severe fever with thrombocytopenia syndrome virus strains and prediction of patient survival based on viral load. J Clin Microbiol. 2014;52: 3325-33.

15. Liu K, Cui N, Fang LQ, Wang BJ, Lu QB, Peng W, et al. Epidemiologic features and environmental risk factors of severe fever with thrombocytopenia syndrome, Xinyang. China PLoS Negl Trop Dis. 2014;8:e2820.

16. Huang $X$, Du Y, Hu X, Ma H, Wang H, You A, et al. Epidemiological and etiological characteristics of fever, thrombocytopenia and leukopenia syndrome in Henan Province, China, 2011-2012. PLoS One. 2014;9:e91166.

17. Ding F, Guan XH, Kang K, Ding SJ, Huang LY, Xing XS, et al. Risk factors for bunyavirus-associated severe Fever with thrombocytopenia syndrome, china. PLoS Negl Trop Dis. 2014;8:e3267.

18. Lu QB, Zhang SY, Cui N, Hu JG, Fan YD, Guo CT, et al. Common adverse events associated with ribavirin therapy for Severe Fever with Thrombocytopenia Syndrome. Antiviral Res. 2015;119:19-22.

19. Gong Z, Gu S, Zhang Y, Sun J, Wu X, Ling F, et al. Probable aerosol transmission of severe fever with thrombocytopenia syndrome virus in southeastern China. Clin Microbiol Infect. 2015;21:1115-20.

20. Jiang XL, Zhang S, Jiang M, Bi ZQ, Liang MF, Ding SJ, et al. A cluster of person-to-person transmission cases caused by SFTS virus in Penglai. China Clin Microbiol Infect. 2015:21:274-9.

21. Kim UJ, Kim DM, Ahn JH, Kang SJ, Jang HC, Park KH, et al. Successful treatment of rapidly progressing severe fever with thrombocytopenia syndrome with neurological complications using intravenous immunoglobulin and corticosteroid. Antivir Ther. 2016;17. [Epub ahead of print]

22. Shimojima M, Fukushi S, Tani H, Taniguchi S, Fukuma A, Saijo M. Combination effects of ribavirin and interferons on severe fever with thrombocytopenia syndrome virus infection. Virol J. 2015;12:181.

23. Shimada S, Posadas-Herrera G, Aoki K, Morita K, Hayasaka D. Therapeutic effect of post-exposure treatment with antiserum on severe fever with thrombocytopenia syndrome (SFTS) in a mouse model of SFTS virus infection. Virology. 2015:482:19-27.

\section{Submit your next manuscript to BioMed Central and we will help you at every step:}

- We accept pre-submission inquiries

- Our selector tool helps you to find the most relevant journal

- We provide round the clock customer support

- Convenient online submission

- Thorough peer review

- Inclusion in PubMed and all major indexing services

- Maximum visibility for your research

Submit your manuscript at www.biomedcentral.com/submit
Biomed Central 